\title{
SUPRASELLAR PITUITARY TUMOUR: UNEXPECTED SURGICAL OUTCOME AND LEARNING LESSONS
}

AUTHORS: Rajan Bhargava* (1) Mohit Kacker (2) Anjali Tiwari (3) Deepti Gupta (4)

AUTHORS AFFILIATIONS: (1) Consultant Otolaryngologist (2) Consultant Neurosurgeon (3) Consultant Pathologist (4) Consultant Pathologist: Regency Hospital, Kanpur

\begin{abstract}
Suprasellar pituitary tumors are commonly operated by Endonasal Endoscopic Approach (EES) with very acceptable morbidity and complication rate. Occasionally tumor morphology or unexpected surgical trauma can lead to worse outcomes. We present a case of pituitary tumor with a large suprasellar cystic component invading the third ventricle who succumbed after an uneventful surgery. The possible reasons and learning thereof is being discussed in this report.
\end{abstract}

Keywords : Suprasellar tumors, EES, Complications

\section{INTRODUCTION}

EES approach to pituitary and anterior skull base lesions is a well established surgical technique. It was initially reported by Jankowski et al(1) more than twenty years ago. This was later popularized by many others including Sethi (2), Carrau et al(3).Extended approaches to suprasellar region were a natural advancement with the similar endonasal surgical corridor Cappabianca et al(4).

We report a case of a 32 year old male with a small enhancing sellar lesion and a large cystic suprasellar component with ensuing hydrocephalus. He had clinical presentation of altered consciousness, left sided limb weakness and bilateral visual loss. He had also undergone a right ventriculoperitoneal (VP) shunt elsewhere which was nonfunctional. An uneventful endoscopic endonasal decompression of tumour and saucerisation of cyst with revision VP shunt on right side was done. Patient showed a good response initially followed by a stormy postoperative course with multiple complications resulting in his death after two weeks. The possible reasons for such a cascade of events are discussed with possible remedial measures.

\section{CASE REPORT}

A 32 year old man presented to us with holocranial headache, left sided limb weakness and bilateral visual loss with left being worse as compared to right. He had consulted many other specialists and had undergone right VP shunt elsewhere which was malpositioned and hence was nonfunctional .His examination revealed that he was arousable but sleepy with a power grade IV on left side. His pupils were semidilated and vision clinically was finger counting on right side at one meter and just finger perception on left side. In view of his clinical condition a decision to operate on him was taken on an urgent basis after getting appropriate workup and radiology. A CECT/MRI revealed small enhancing solid(sellar) and large cystic suprasellar lesion with obstructive

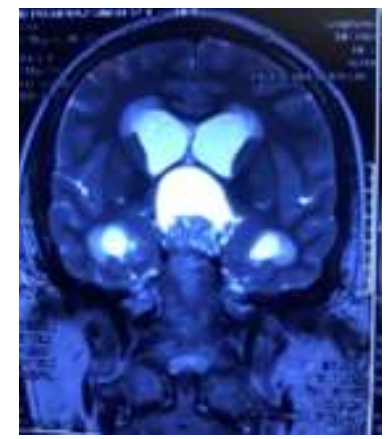

Figure 1

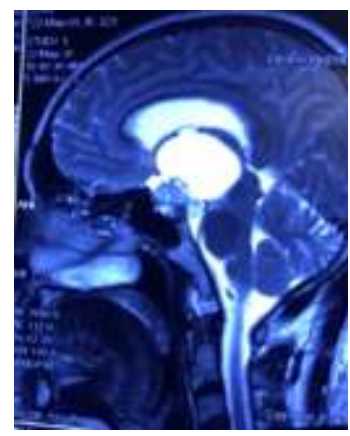

Figure 2 
hydrocephalus suggestive of a either a cystic solid craniopharyngioma or a pituitary tumour with a suprasellar cyst Fig(1\&2).

A post shunt $C T$ showed malpositioned catheter which was non-functioning as mentioned earlier. Patient underwent removal of old VP shunt and placement of new Chabra shunt on the right side via same incision. This was done since ventricular end was short and valve of the shunt was blocked with blood clot and brain matter. At the same time, patient underwent endoscopic endonasal transsphenoidal tumour decompression. The solid, sellar portion of tumour was curetted which was brown,firm and non suckable. Thereafter, thin suprasellar cyst wall was saucerised and floor of third ventricle visualised. Repair of sellar floor was done with fat, fascia lata, Hadad flap and fibrin glue.

The patient did well in the initial postop phase with improved consciousness but with slightly increased urine output. Overnight, patient became very sleepy with increasing urine outputof $2700 \mathrm{ml}$ in 10 hours and a postop CT was advised. This revealed air and fluid in the area of suprasellar cyst, a well functioning right shunt and persistent hydrocephalus in the left ventricular system. It was decided to do a left VP shunt despite worsening of patient's symptoms who developed deterioration in sensorium, hyperpyrexia(105F)and severe tachycardia.A left VP shunt was done but no improvement in symptoms was seen with worsening sensorium and increasing urine output neccesasitating desmopressin administration. The patient could not tolerate extubation and had a generalised tonic clonic seizureand had to be reventilated. He never regained consciousness and had to be tracheostomized for ventilatory support but unfortunately died two weeks later due to chest sepsis.The histopathology of the tumour was pituitary adenoma which was surprising as radiological features pointed a diagnosis towards craniopharyngioma.The histopathology revealed a richly vascular tumour composed of moderately pleomorphic cells disposed in papillae, sheets, pseudorosettes and pseudoglandular pattern. Tumour cells had round to oval nucleus, coarsely granular chromatin, occasional prominent nucleoli and moderate amount of eosinophilic granular cytoplasm.Areas of haemorrhage and cystic change were seen.On IHC tumour showed strong positivity for synaptophysin and PCK whereas EMA, LCA, prolactin, GH and GFAP were negative. $\mathrm{KI} 67$ showed low proliferative index less than $1 \%$.Hence the diagnosis of pituitary adenoma was made.
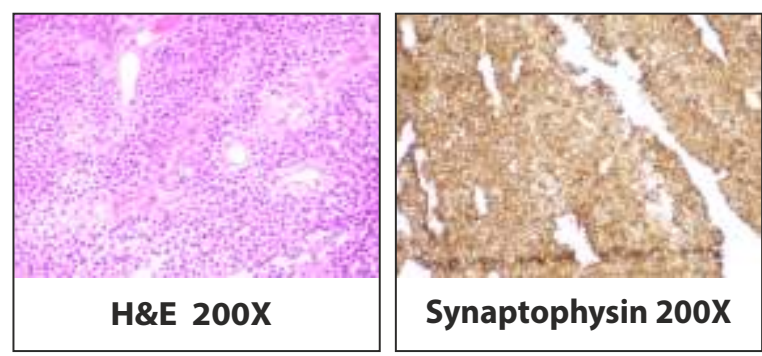

\section{DISCUSSION}

EES for lesions of pituitary and anterior skull base is an established surgical practice all over the world with an acceptable morbidity and mortality rate. A mortality rate of less than $1 \%$ has been the norm in most studies, Cappabianca et al (5).The complications arising out of such surgeries have been anatomically classified being nasofacial, sphenoidal, sellar, suprasellar, parasellar and endocrine, Cappabianca et al (5).The outcomes are dependent on both surgeon's hands and specific tumour features. A learning curve is definitely present in the overall outcome, Soliman et al(6). Our patient with a small sellar component and a large suprasellar cyst with invasion of third ventricle behaved more like a craniopharyngioma. With more than 100 such cases operated by our centre we felt fully equipped to take on this surgery. The surgical goals appeared to be accomplished with reasonable tumour decompression, uncapping of cyst and revision of shunt. His 
stormy postoperative phase with an initial recovery was a surprise to us. We are sharing our thoughts after doing a retrospective analysis of the course of the patient as a learning exercise.

Endoscopic decapping of large suprasellar cyst would inevitably lead to the entry of air and blood in the dead space in postoperative phase. This could have resulted in pressure on bilateral foramen of Monroe and persistent hydrocephalus. Hence we could have been safer in shunting both sides rather than believing that tumour decompression alone would suffice to improve hydrocephalus. The left sided shunt done as an after thought did not help because patient had deteriorated considerably by that time.Surprisingly literature is fairly silent on this.

Secondly our patient developed generalised tonic clonic seizures with reasonable biochemical parameters. Although EES is minimally invasive but where surgical manipulation is more than usual a prophylactic anticonvulsant can be considered.

Thirdly hyperpyrexia raises the possibility of acute hypothalamic injury during surgery especially in the manipulation of surasellar component.Literature deals with hypothalamic injury more in relation to craniopharyngioma rather than pituitary surgery as such. The situation in our patient was more like a craniopharyngioma rather than a classical pituitary tumour as interpreted from radiology. Tumour origin site along the pituitaryhypothalamic axis, invasion of third ventricleare a major determinant of hypothalamic injury and poor outcome with surgical mortality upto $21.5 \%$, Prieto et al (7). The shape of the suprasellar lesion and distortion of pituitary stalk on MRI were other pointers towards a likely hypothalamic injury during surgery, Prieto et al (7).

Finally a case has to made for prolonged ventilation if required although it raises the cost of care. In a study by Rock et al(8) unfavourable events such as prolonged ventilation were under reported.

Such lesions not only require meticulous surgical dissection but also an in depth understanding of complex anatomy and attendant risk factors which may hamper a good postoperative result. A case is also to be made of fairly exacting postoperative care to improve results.

\section{CONCLUSION}

EES although is an established surgical method for anterior skull base lesions but complications related are also not unknown. They are usually very safe but an occasional mortality can dampen the enthusiasm of the oprative team. Learning curve, tumour morphology, extent of resection, unfavourable anatomy and postoperative care play a role in overall surgical outcome.

\section{BIBLIOGRAPHY}

(1) Jankowski R,Auque J,Simon C,Marchal JC,Nepner H,WayoffM, Endoscopic Pituitary tumour Surgery.Laryngoscope 1992;102(2):198-202

(2) Sethi DS,Prem K. Pillay, Endoscopic Pituitary Surgery-, a minimally invasive technique.Am J Rhinol 1996;10(3):141147

(3) Carrau RL, Jho ND,Koy, Transnasal -transsphenoidal endoscopic suegery of the pituitary gland. Laryngoscope1996;106(7):914-918

(4) Cappabianca P, Frank G,Pasquini E,et al . Extended endonasal trans-sphenoidal approaches to the suprasellar region, planum sphenoidale and clivus. In de Divitiis E, Cappabianca P, eds. Endoscopic Endonasal Transsphenoidal Surgery. Wein :Springer -Verlag;2003 
(5) Cappabianca P,Cavallo LM,De Divitiis E, , Surgical complications associated with the endoscopic endonasal trans sphenoidal approach for pituitary tumours. J. Neurosurg 2002;97:293-298

(6) Soliman MAR, Eaton S, Alkhamees AF et al ,Challenges, Learning Curve,and Safety of Endoscopic Endonasal Surgery of Sellar-Suprasellar Lesions in a Community Hospital World Neurosurgery 2020;138:940-954

(7) Prieto Ruth, Pascual JM, Barrios Laura, Letter to editor,Assessment of Postoperative complications in craniopharyngioma patients:An approach based on the heterogenous tumour-hypothalamus axis. Surgical Neurology International 2020;11(47)1-3

(8) Rock AK, Dincer A, Carr MT, et al Outcomes after craniotomy for resection of craniopharyngiomas in adults :Analysis of the national surgical quality improvement program(NSQID). J Neurooncol 2019;144:117-25

Conflict of interest: We have no conflict of interest to declare.

Source of support: Nil

\section{*Corresponding Author}

Dr Rajan Bhargava

Consultant ENT surgeon

Regency Hospital

Kanpur

Email id -rishabhbha@gmail.com 